\section{A mask facilitating multiple analyses by micro-immunoelectrophoresis}

J. G. FEINBERG ${ }^{1}$, K. L. G. GOLDSMITH, and R. A. KEKWICK From the Beecham Research Laboratories, M.R.C. Blood Group Reference Laboratory, and the Lister Institute, London

The technique of immunoelectrophoresis in agar gel was devised by Grabar and Williams (1953) and subsequently Scheidegger (1955) introduced a micro-method based on the same principles. Whichever procedure is used, wells for the antigen and slots for the antiserum are cut or moulded in the agar. Cutting wells and slots in agar is a tedious process and one that is difficult to perform reproducibly within the fine limits required, particularly when the micro-method is employed. Moreover, if the preparations are finally stained, the dyes employed tend to adhere along the margins of the slots and wells, whether these have been cut or moulded.

Recently Feinberg (1964) described a plastic mask for use in immuno-diffusion experiments of the Ouchterlony-Elek type. In this paper the application of a mask for facilitating micro-immuneolectrophoresis experiments is described.

\section{MATERIALS}

In Fig. 1 the details are given of a mask, the dimensions of which have been found most suitable after testing a number of slightly differing designs. The mask $\left(5 \frac{1}{2} \times\right.$ $3 \frac{1}{2}$ in.; $14 \times 9 \mathrm{~cm}$.) was cut from polyvinyl-chloride sheet 0.020 in. (ca. $0.5 \mathrm{~mm}$.) thick. This material adheres firmly to agar when applied as described below and there is no creeping of antigen solution or antiserum between the upper surface of the agar and the lower surface of the mask.

Between the antiserum slots which are 3 in. $(7.5 \mathrm{~cm})$ long, $3 / 32$ in. ( $2 \mathrm{~mm}$.) wide, and $5 / 8$ in. $(16 \mathrm{~mm}$.) apart, two series of antigen holes are drilled, each series on a line situated 1 in. $(2.5 \mathrm{~cm}$.) inwards from the lines joining the ends of the slots. In one series the hole is $3 / 32$ in. and in the other $1 / 8$ in. diameter, so allowing an approximately twofold variation in the amount of antigen that can be applied. The inward displacement of the holes is to compensate for endosmosis effects during electrophoresis.

In addition to the masks, plates 6 in. $\times 5$ in. $(15 \times$ $12.5 \mathrm{~cm}$.) cut from good quality $12 \mathrm{oz}$. window glass are required.

\section{PROCEDURE}

Into a dish of suitable dimensions is poured sufficient molten $1 \%$ agar in buffer to form a layer about $3 \mathrm{~mm}$.

'Present address: Miles Laboratories Ltd., Stoke Court, Stoke Poges, Slough, Bucks.

Received for publication 10 April 1964.

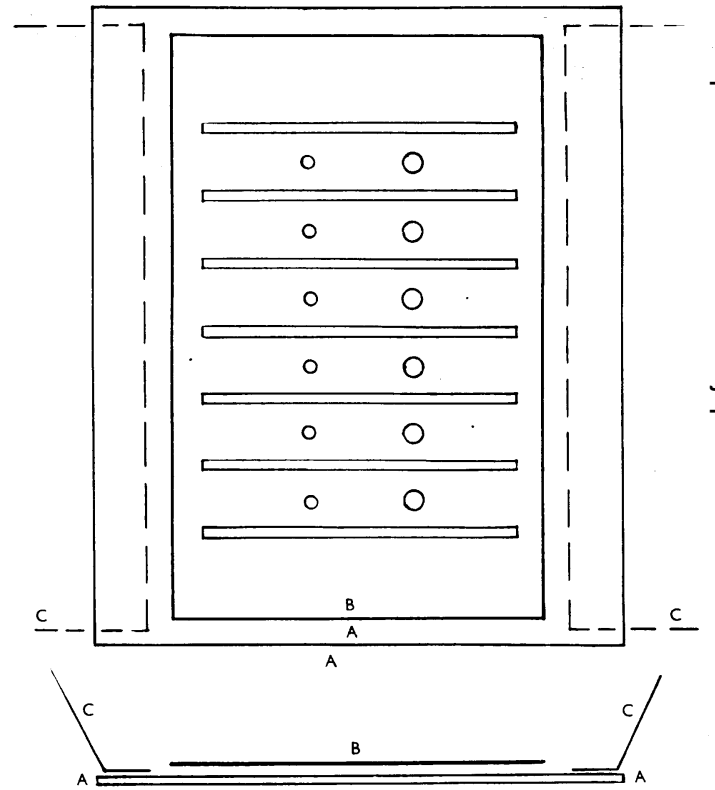

FIG. 1. Plan and section showing arrangement of glass $\overrightarrow{\mathscr{\leftrightarrow}}$ plate, polyvinylchloride former, and filter paper wicks in ${ }^{\ddagger}$ electrophoresis assembly.

$A$, glass plate; $B$, polyvinylchloride former, $C$, filter paper wicks.

thick; when this has set it provides a flat horizontal $\frac{\partial}{\circ}$ surface on which the glass plate is laid. Wicks of What- $\varrho$ man no. 3 filter paper, partially folded to provide $a_{0} \overrightarrow{0}$ narrow transverse flap, are placed with the flap in contact 3 with the glass along the entire length of the longer sides of the glass plate and overlapping the edge by about $1 \mathrm{~cm}$. (Fig. 1). Sufficient molten agar in buffer is then poured over the whole area to give a depth of about $3 \mathrm{~mm}$. overo the glass plate. While this agar is still liquid the mask is gently placed on the surface without trapping any air 3 bubbles between the mask and the agar. When the agari is set the mask adheres firmly to the agar surface and the wicks are also sealed to the glass plate.

The agar is then cut with a sharp knife along the edges 3 of the glass plate and the plate carrying the upper film of agar, mask and wicks, is lifted from the lower film of agar and transferred bodily to a suitable electrophoresis chamber.

The antigen holes on that side of the mask towards 0 which electrophoretic migration will occur, usually then anode side, are just filled with antigen dissolved in or dialysed against buffer, and $45 \mathrm{~min}$. allowed for the antigen to diffuse into the agar. A potential is there applied from a rectifier, sufficient to produce a currented of $2 \mathrm{~mA}$ per $\mathrm{cm}$. width of agar gel, that is, a total current $t^{+}$ in this instance of $30 \mathrm{~mA}$. Under these conditions, ifo electrophoresis is allowed to proceed for five hours the proteins of a sample of human plasma will be distributed across the entire length of the 3 in. path available between $\vec{D}$ a pair of antiserum slots in the mask. 
A

B C

$E$

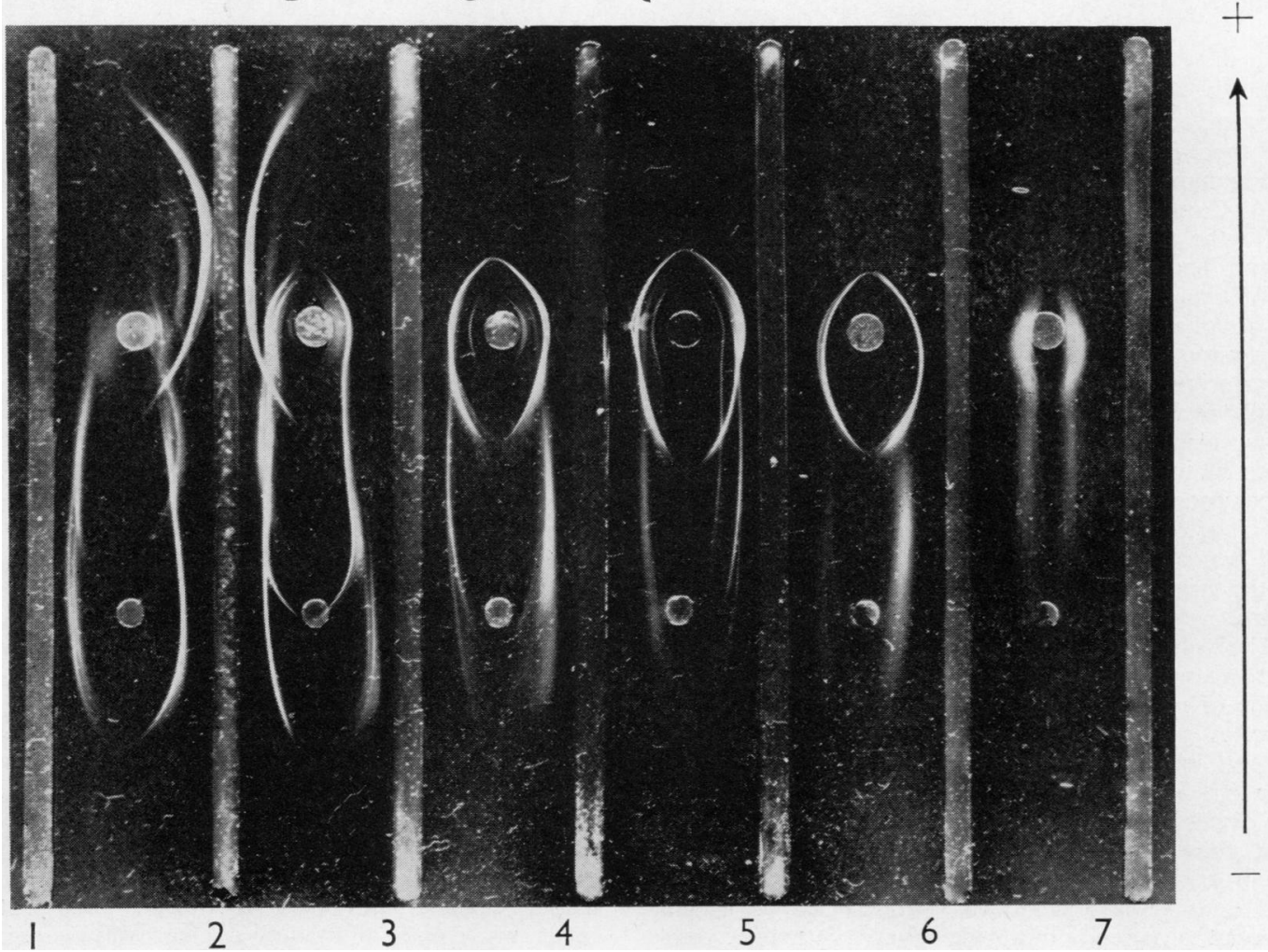

FIG. 2. Photograph of a plate taken after 90 hours' development with antiserum at $4^{\circ} \mathrm{C}$.

Antigens: $A$, normal human plasma $(1 \%) ; B$, macroglobulinaemia serum $(1.5 \%)$; $C$, fraction from normal human plasma containing $19 S \alpha$-and $19 S-\gamma$ globulins; $60 \%$ of total protein $19 S$ globulin $(1 \cdot 5 \%) ; \mathrm{D}$, pellet from $C$ obtained by preparative ultracentrifuging; $90 \%$ of total protein $19 S$ globulin $(1.5 \%) ; E$, chromatographic fraction from D about $90 \% 19 S \alpha$-globulin $(0 \cdot 7 \%) ; F$, chromatographic fraction from D mostly $19 S \gamma-g l o b u l i n$ but showing a deleterious effect of the chromatographic procedure on the character of the $19 S \gamma-g l o b u l i n(0 \cdot 3 \%)$.

Figures in brackets indicate total protein content of antigen solution applied.

Antisera: all raised in rabbit; 96 against whole human plasma, 43 and 44 using fraction $D$ for immunizing. Channels 1, 3, 5, 7 filled with antiserum 44; channels 4 and 6 filled with antiserum 43; channel 2 filled with antiserum 96.

At the end of this period the plate is removed and, after the wicks have been cut off, is placed in a suitable dish. The slots in the mask are then filled with appropriate antisera, the dish is covered and the whole assembly left to allow precipitin arcs to develop. Some bands usually appear overnight but 48 to 96 hours may be necessary for their full development, a process which can of course be observed through the mask. The mask is then lifted from the agar film, and the preparation may be photographed or stained by established procedures.

A photograph of a developed plate is shown (Fig. 2).

COMMENT

The electrophoresis and development procedures described have usually been carried out in a cold room at about $4^{\circ} \mathrm{C}$. In these circumstances it is convenient to pour a plate and leave it overnight at $4^{\circ} \mathrm{C}$. to cool thoroughly before it is used. The experiments can be done entirely at room temperature if this is preferred, or the electrophoresis may be conducted at room temperature and the plate left to develop at $4^{\circ} \mathrm{C}$. The precipitin arcs are more sharply defined if they are allowed to develop at $4^{\circ} \mathrm{C}$., though they form a little more slowly under these conditions than at room temperature.

Higher concentrations of agar than $1 \%$ tend to increase the time required for the development of the arcs, but $1 \%$ agar is the lowest concentration that is satisfactory for use at room temperature.

Both veronal and glycine- $\mathrm{NaCl}$ buffers, $p \mathrm{H} 8.5$ ionic strength 0.05 , have been used satisfactorily with Difco agar or Ionagar no. 2 (Oxoid). 


\section{Book reviews}

VIRUSES, NUCLEIC ACIDS AND CANCER A Collection of Papers presented at the 17th Annual Symposium on Fundamental Cancer Research, 1963. Edited by R. W. Cumley et al. (Pp. 659; illustrated. \$16.-) Published for the University of Texas, M.D. Anderson Hospital and Tumour Institute. Baltimore: The Williams and Wilkins Co.

This volume of 650 pages includes the papers and discussions presented at the Seventeenth Annual Symposium on Fundamental Cancer Research. The emphasis was upon the viral aspects of tumours and "the facts, ideas, theories and discussions presented were intended to converge the various research disciplines upon a common goal ... the solution of the human cancer problem'. Although no solution will be found the papers and discussions contained in this book contribute much to the viral concepts of oncogenesis as a working hypothesis.

The volume is divided into eight main sections. The first two are devoted to papers on the structure of viruses, mode of replication, and biochemistry of virus-infected cells. A series of seven papers on viral nucleic acids follows, which contains a mass of up-to-date information. The biological properties of the avian tumour viruses are discussed in a further section, from which it is clear that these viruses consist of a number of distinct strains each of which has the capacity to induce a varied and multiple host response. Two further sections are devoted to papers with a wide variety of interest: viruses and interferon, interference and cell division, the biological aspects of tumour induction, the oncogenicity of human adenovirus, and an account of the malignant lymphoma recently recognized in Africa. In addition are included several comprehensive reviews on mouse leukaemia by Ludwick Gross, 'Newer and older

A mask facilitating multiple analyses by micro-immunoelectrophoresis - concluded.

The method has obvious advantages: six antigens may be examined simultaneously, or seven antisera tested simultaneously against the same antigen using very small quantities of reagents. In addition it has proved to be most useful in applying the Osserman (1960) procedure for identifying, in mixtures, the individual antigens responsible for the production of specific precipitin lines.

We wish to thank Mr. B. Jordan for his excellent technical assistance in developing this procedure.

\section{REFERENCES}

Feinberg, J. G. (1964). Nature (Lond.), 201, 631

Grabar, P., and Williams, C. A. (1953). Biochim. biophys. Acta (Amst.), $10,193$.

Osserman, E. F. (1960). J. Immunol., 84, 93.

Scheidegger, J. J. (1955). Int. Arch. Allergy, 7, 103. viruses in mammalian malignancy' by Dr. Maurice $\frac{\bar{\rho}}{-}$ Hilleman, and a final summary by Dr. Frank Horsfalk on 'The role of viruses in relation to cancer in animals్ and man'.

This book has been published at a most opportune $\overrightarrow{0}$ time when interest in the role of viruses and tumours is growing every day. It is thoroughly recommended as an excellent guide to one of the most fascinating and import ant branches of modern medicine. It is well produced? well illustrated, and contains a comprehensive biblio $\vec{\nabla}$ graphy with each section.

J. A. DUDGEON

MODERN TRENDS IN IMMUNOLOGY I. Edited by Roberto Cruickshank. (Pp. vii + 263; illustrated. 65s.) London :윽 Butterworth. 1963.

This is a collection of 11 essays on topics in immunology. They fall into three groups. Those in the first group are concerned with the properties of antigen, of antibodies and the mechanisms of, and techniques for observing antigen-antibody interaction. Each of these is a concised and competent statement of current knowledge. In thenext section can be grouped five articles on immunity. The first of these deals with natural immunity (natural ${ }^{+}$ resistance) and covers the role of cells of the reticulo-endothelial system, of serum factors and of the non specific effects which, for example, often follow injection of endotoxin. Acquired immunity is considered in three⿳亠丷厂 essays dealing with bacterial, viral, and protozoalo infections. This is followed by an article on immunization in which the present status of immunization procedures $\overrightarrow{\bar{\sigma}}$ for major diseases is summarized. The final three articles 3 discuss neatly and compactly hypersensitivity, auto-? immunity, and transplantation immunology.

Immunology is a rapidly expanding subject so that it is not hard to find topics which are barely mentioned However, the topics chosen do give a comprehensive cover and illustrate clearly that workers in many different disciplines are involved in some aspect or other of immunological research. The biochemist will admire the application of techniques which are so neatly revealing the structure of antibodies. The physician will be gratefuB for the succinct statement on immunization agains $\square$ major diseases, and the public health authorities should ponder the remarks about cholera, considered in the essay on bacterial infections. The biologist will be af home in all the articles.

To the reviewer, 'modern trends' indicates that theN topics chosen are the subject of active research and thaf the writer should discuss such aspects and indicate possible future advances. Most of the articles go a lon $\$$ way towards this but some, such as the discussions orm acquired immunity to bacterial and viral infection, would? suggest, wrongly, that these are more moribund topics. T

The volume is certain to attract a wide audience. The format is acceptable, few typographical errors werd noticed, and the price, 65 shillings, is moderate.

G. L. ADA 